Revista Científica de FAREM-Estelí

Medio ambiente, tecnología y desarrollo humano

Año 10 | Núm. 38| Abril-junio, 2021

ISSN: 2305-5790

https://rcientificaesteli.unan.edu.ni

DOI: https://doi.org/10.5377/farem.v0i38.11939

\section{Estrategias comunitarias de prevención de la desnutrición en niños de 0-5 años en el puesto de Salud el Carmen, sector 9, municipio de San Juan del Río Coco, Nicaragua, 2019}

\section{Community strategies for the prevention of malnutrition in children aged 0-5 years at the El Carmen health post, sector 9, municipality of San Juan del Río Coco, Nicaragua, 2019}

\section{Linda Celeste Álvarez Martínez}

Facultad Regional Multidisciplinaria, Estelí. UNAN-Managua/FAREM-Estelí, Nicaragua

linddaceleste_96@gmail.com

\section{Samuel Antonio Centeno Córdoba}

Facultad Regional Multidisciplinaria, Estelí. UNAN-Managua/FAREM-Estelí, Nicaragua

scentenoc51@gmail.com

\section{Gabriela Areli Gil Valenzuela}

Facultad Regional Multidisciplinaria, Estelí. UNAN-Managua/FAREM-Estelí,

Nicaragua

gabrielareli@yahoo.es

\section{Beverly Castillo Herrera}

Doctora en Ciencias Sociales. Docente titular con doctorado (TD). FAREM-Estelí, UNAN-Managua.

beverly.castillo@yahoo.com

\section{RESUMEN}

Históricamente, en las comunidades del municipio de San Juan del Río Coco, se han presentado un deterioro en el estado nutricional de la niñez en edad de 0 a 5 años, según datos del Ministerio de Salud, afectando aproximadamente al $60 \%$ de este grupo etario. Por ello, este estudio busca determinar el estado de desnutrición en la niñez de 0-5 años que son atendidos en el puesto de salud el Carmen, para aportar al diseño de una estrategia comunitaria enfocada en la prevención de la desnutrición. Según el enfoque filosófico es una investigación cuantitativa. Se aplicaron 214 encuestas a las madres de niños-as afectados, entrevistas al personal de salud, líderes comunitarios y familia. La hipótesis se cumple al demostrar que existe una relación inversamente proporcional entre prácticas alimentarias y hábitos de higiene con la desnutrición. Se propone una estrategia comunitaria que pretende contribuir a la erradicación de la desnutrición en la niñez de cero a cinco años en el sector el Carmen, San Juan del rio coco, Nicaragua. Para su ejecución se proponen las siguientes acciones: promover la organización y participación de los diferentes actores sociales, propiciar el acceso de las familias a alimentos nutritivos, sanos y suficientes, y favorecer la sensibilización de la población sobre las consecuencias de la desnutrición en el desarrollo de la niñez de 0-5 años.
RECIBIDO

$24 / 02 / 2021$

ACEPTADO

15/062021

\section{PALABRAS CLAVE}

Desnutrición; estado nutricional; hábitos de higiene; prácticas alimentarias; estrategias comunitarias. 


\section{ABSTRACT}

Historically, in the communities of the municipality of San Juan del Río Coco, there has been a deterioration in the nutritional status of children aged 0-5 years, according to data from the Ministry of Health, affecting approximately $60 \%$ of this age group. Therefore, this study seeks to determine the state of malnutrition in children aged 0-5 years who are treated at the El Carmen health post, in order to contribute to the design of a community strategy focused on the prevention of malnutrition. According to the philosophical approach, it is a quantitative research. Two hundred and fourteen surveys were applied to mothers of affected children, interviews to health personnel, community leaders and family. The hypothesis is fulfilled by demonstrating that there is an inversely proportional relationship between food practices and hygiene habits and malnutrition. A community strategy is proposed that aims to contribute to the eradication of malnutrition in children from zero to five years of age in the sector of El Carmen, San Juan del Rio Coco, Nicaragua. The following actions are proposed for its implementation: promote the organization and participation of the different social actors, promote the access of families to nutritious, healthy and sufficient food, and promote the awareness of the population about the consequences of malnutrition in the development of children 0-5 years old.

\section{KEYWORDS}

Malnutrition; nutritional status; hygiene habits; food practices; community strategies. 


\section{INTRODUCCIÓN}

El Puesto de Salud El Carmen se encuentra ubicado en la Comunidad de su mismo nombre, la cual pertenece al municipio de San Juan del Río Coco en el Departamento de Madriz. Este Puesto de Salud brinda atención a la población de cuatro comunidades incluyendo la comunidad sede. Históricamente, en estas comunidades se ha presentado de manera casi generalizada un deterioro en el estado nutricional de la niñez en edad de 0 a 5 años de este territorio, afectando aproximadamente al $60 \%$ del total de la niñez en esta edad.

Estableciendo una relación comparativa entre los datos oficiales del Ministerio de Salud (MINSA), para el año 2018 el porcentaje de desnutrición aguda a nivel nacional alcanzaba un $12.8 \%$ de la niñez menor de cinco años, y en el caso particular del municipio de San Juan de Río Coco este porcentaje se elevaba hasta el $60 \%$ aproximadamente, evidenciando un serio problema de salud como es la desnutrición infantil, que afecta a este sector tan importante de la población de ese municipio convirtiéndose ya en un problema crónico al cual urge dar respuesta.

El MINSA, garante de los derechos de los nicaragüenses a la salud y a estar protegidos contra el hambre, considera el combate a la desnutrición crónica como uno de los ejes centrales de sus políticas públicas; por tanto, a partir del año 2008 se definió el uso del indicador de desnutrición crónica en niños menores de 5 años, incluidos en la matriz de seguimiento al logro de los Objetivos del Desarrollo Del Milenio (ODS).

La desnutrición en sus diversas formas es la más común de las enfermedades, según datos recientes de peso y talla de niños y niñas de la zona en estudio, la desnutrición crónica prevalece por encima de la desnutrición aguda; tal situación hace pensar que existen prácticas deficientes de las diversas estrategias y acciones comunitarias implementadas por el MINSA, que conllevan a secuelas y daños irreversibles en la población menor de 5 años del municipio de San Juan de Río Coco.

Las causas de la desnutrición a nivel global son multifactoriales y muchas de ellas se pueden extrapolarse al territorio en estudio, por ejemplo la incidencia del bajo nivel socioeconómico en dichas familias las cuales se caracterizan por ser numerosas, sufrir de hacinamiento y tener bajo nivel académico, su principal actividad económica es la agricultura de subsistencia y el cultivo del café, el cual depende fundamentalmente del precio del mercado internacional y de las condiciones climáticas, por lo que no se puede hablar fácilmente de réditos seguros en este rubro, sobre todo para el campesinado pobre. No es casual que para solventar el déficit financiero recurran a la práctica de omitir uno o más tiempos de la dieta básica. 
La desnutrición se acompaña por la deficiencia de vitaminas y minerales en la alimentación que causan en estos niños bajo rendimiento escolar, disminución de coeficiente intelectual, problemas en el aprendizaje, disminución en la retención y memoria, escaso desarrollo muscular. Esta situación es de mayor prevalencia en áreas rurales como la zona de estudio, por las condiciones socioeconómicas precarias en las que viven, porcentaje de analfabetismo y escasez de productos alimenticios que contengan los nutrientes necesarios para el desarrollo y crecimiento del niño e incluso por la falta de conocimiento acerca de dichos alimentos que quizás tienen a mano, pero no saben el aporte nutritivo que le puede proporcionar al niño.

La incidencia de los factores socioculturales son también determinantes en la prevalencia del problema de la desnutrición en el Municipio de San Juan de Río Coco, donde es muy común encontrarse con algunas prácticas que tienen connotaciones de género y generacional, como la inequidad en la distribución de los alimentos dando prioridad a los hombres y a las y los adultos, sustentando esta práctica en conceptos tradicionales no positivos en el que niñez es sinónimo de pequeño y por tanto lo que se le asigne debe ser poco.

Si bien es cierto, que la desnutrición no se hereda se asegura que cuando ésta se mantiene por generaciones consecutivas, produce factores modificadores que se transmiten de madres y padres a hijos y a esto se le denomina herencia social, por lo que si agregado a antecedentes de desnutrición de una madre, ésta no recibe el cuidado prenatal requerido, seguramente el hijo podrá estar sometido a restricción del crecimiento intrauterino, bajo peso al nacer o anemia, entre otras consecuencias.

Otro elemento importante que está vinculado al aspecto sociocultural y que tiene gran impacto en el estado nutricional de la niñez es la lactancia materna exclusiva durante los seis primeros meses de vida, la cual de no cumplirse genera la no transmisión de anticuerpos y por tanto niños y niñas con mayor número de hospitalizaciones, así como la utilización de sucedáneos de la lactancia materna y ablactación temprana.

Además, existen dos nuevos factores que intervienen en el fenómeno de la desnutrición infantil, siendo éstos: los malos hábitos higiénicos en los hogares, que propician enfermedades recurrentes sobre todo en la niñez y la no incorporación de alimentos variados para asegurar una dieta balanceada, como un elemento clave para la salud humana.

Esta investigación considera tres ejes teóricos: 1) Nutrición y desnutrición, 2) Hábitos alimenticios y de higiene; y 3) Estrategias comunitarias.

Soriano del Castillo (2011, pág. 23) define la desnutrición como el conjunto de procesos mediante los cuales el organismo vivo, en este caso el ser humano, utiliza, transforma e incorpora una serie de sustancias que 
recibe del mundo exterior y que forman parte de los alimentos con objeto de suministrar energía, construir y reparar estructuras orgánicas, así como regular los procesos biológicos.

El concepto de estado Nutricional se expresa como el resultado de comparar el peso obtenido con relación a la talla y a la edad del niño o niña, con los límites establecidos como normales para ese peso, talla y edad, según los nuevos patrones de crecimiento infantil de la OMS. La medición del estado nutricional, se realiza la primera vez que llega el niño o la niña en el año calendario. (MINSA, 2009, pág. 66)

Para la UNICEF (2011, pág. 9) la desnutrición crónica es un problema de mayor magnitud en cuanto al número de niños afectados, es a veces invisible y recibe menor atención. El retraso en el crecimiento puede comenzar antes de nacer, cuando el niño aún está en el útero de su madre. Si no se actúa durante el embarazo y antes de que el niño y niña cumpla los dos años de edad, las consecuencias son irreversibles y se harán sentir durante el resto de su vida

La desnutrición es una condición patológica que puede ser reversible o no, ocasionada por la carencia de múltiples nutrientes, derivada de un desequilibrio provocado por un insuficiente aporte de energía, un gasto excesivo, o la combinación de ambos, que afecta en cualquier etapa del ciclo vital, en especial a lactantes y niños. Para la OMS la desnutrición se considera un IMC en niños igual o por debajo de 17,4. (Bamba, Espinoza, \& Fajardo, 2010)

El Ministerio de Salud de Nicaragua (MINSA, 2012) afirma que está demostrado que entre los 6 a 24 meses de vida se instala la desnutrición crónica, como consecuencia ocurren pérdidas irreparables en la talla de los niños y niñas, su capacidad intelectual y sistema inmunológico. Por tanto, la prevención de la desnutrición debe ser un proceso que inicia desde el embarazo, haciendo énfasis en los cuidados en los primeros 6 meses de vida del niño y niña y hasta los dos años

En la desnutrición influyen los hábitos alimentarios que son el conjunto de costumbres que condicionan la forma como los individuos o grupos seleccionan, preparan y consumen los alimentos influida por la disponibilidad de estos, el nivel de educación alimentaria y el acceso a los mismo. (Ruiz Hurtado, 2018, pág. 36)

Así mismo, la higiene alimentaria es indispensable mantenerla porque son el conjunto de condiciones y medidas que deben estar presentes en todas las etapas de producción, almacenamiento, transformación, transporte, conservación y cocinado doméstico del alimento, para garantizar la salubridad de los alimentos (OMS, 2018, pág. 1). De esta manera, los hábitos de higiene se encuentran vinculados con el estilo de vida que se 
define como "el conjunto de pautas de conducta y hábitos cotidianos de una persona (Barahona \& Barahona, 2012, pág. 8)

Una estrategia comunitaria se define como la gama y combinación de actividades y decisiones que los pueblos y comunidades realizan y optan para lograr sus objetivos en relación a medios de vida y sobre todo en dependencias de las necesidades y principales carencias o problemas sociales previamente identificados (Jansen, Rodriguez, Damon, \& Pender, 2003, pág. 32)

En el estudio que se realizó se determina el estado de desnutrición de niñas y niños de 0 a 5 años que son atendidos en un Centro de Salud Rural del municipio de San Juan del Río Coco, a fin de obtener la información necesaria para proponer una estrategia comunitaria que contribuya a disminuir la incidencia de esta problemática con el involucramiento directo de la población para un adecuado desarrollo nutricional de la niñez de la localidad.

Los hallazgos de este estudio servirán de insumos al Ministerio de Salud y demás entidades del Estado garantes de derecho encargados de la vigilancia y acompañamiento a las familias para el adecuado crecimiento y desarrollo de la niñez, para la toma de decisiones y lograr mejorar el estado nutricional de la niñez menor de cinco años cumpliendo la meta 2.2 del Segundo ODS, que propone "De aquí a 2030, poner fin a todas las formas de malnutrición. Este tema investigativo brinda un aporte científico a Universidad Nacional Autónoma de Nicaragua - FAREM-Estelí, considerando está regida bajo los lineamientos investigativos siendo pionera en la elaboración de una estrategia comunitaria orientada a esta problemática en la comunidad El Carmen en el municipio de San Juan del Río Coco y además, no solo se trata de la descripción de la población y sus problemáticas sino que tiene un enfoque analítico-propositivo que conlleva a deducir que a menor calidad de las prácticas alimentarias y de hábitos de higiene, mayor es la incidencia de desnutrición en niños de 0 a 5 años

\section{MATERIALES Y MÉTODOS}

La presente investigación según el enfoque filosófico es una Investigación Cuantitativa, porque analiza la relación entre las variables y los nexos existentes entre el estado nutricional y los hábitos alimentarios y de higiene.

Según el uso del conocimiento es una Investigación Aplicada, porque se basa en una necesidad social práctica y sugiere estrategias para resolverla, es decir, busca la aplicación o utilización de conocimientos, desde una o varias áreas especializadas, con el propósito de implementarlos de forma práctica para 
satisfacer necesidades concretas, proporcionando una solución a problemas del sector social o productivo. (Vargas Cordero, 2011, pág. 32)

La población de esta investigación está conformada por 478 niños de 0 a 5 años, que han sido valorados con desnutrición por personal médico del Centro de Salud El Carmen, San Juan de Rio Coco. Se calcula el tamaño de la muestra utilizando la formula estadística para para población finita, y se obtiene una muestra de 214 niños, de 0 a 5 años del Centro de El Carmen, sector 9, del municipio de San Juan de Río Coco.

El tipo de muestreo es muestreo aleatorio simple, o muestreo probabilístico al azar, puesto que todos tienen la misma probabilidad de ser seleccionados.

Se aplicaron 214 encuestas a la familia de los niños y niñas en situación de desnutrición que han sido atendidos en el puesto de salud El Carmen del municipio de San Juan del Rio Coco. Este instrumento abarca seis ítems: características socios demográficos, evaluación de la vivienda y servicios básicos, atención y tratamiento del puesto de salud, hábitos alimentarios y nutricionales, hábitos de higiene, y atención de brigadistas de salud.

Se realizaron entrevistas semi-estructuradas al personal médico brigadistas y familias de los niños y las niñas en situación de desnutrición; en general a unas 30 personas. Estas entrevistas ayudan a sostener el análisis de los datos estadísticos y permite obtener información documental para el diseño de la propuesta de estrategia comunitaria. La aplicación de los instrumentos fue por consentimiento informado.

El análisis estadístico se realizó con el programa SPSS, versión 23, se diseñaron tablas de frecuencia para el análisis, el diseño fue modificado en Microsoft Excel 2010 para mejorar el aspecto visual de las gráficas y facilitar su comprensión. El informe fue realizado en Microsoft Word 2010, donde se trasladaron las gráficas, tablas, se realizó análisis de las mismas y la organización del documento final.

La evaluación del estado nutricional se realizó en las comunidades de El Carmen, Wali, Varillal Arriba. Para el análisis de las variables cuantitativas se aplicó una escala de Likert para medir nivel la desnutrición en niños de 0-5 años en el puesto de Salud el Carmen, sector 9, municipio de San Juan del Rio Coco. 


\section{RESULTADOS}

\section{Caracterización del puesto de salud de la comunidad El Carmen}

El sector 9, perteneciente al puesto de salud ubicado en la comunidad "El Carmen" ", que se localiza a unos 16 kilómetros al norte del municipio de san Juan del Rio Coco es encargada de la atención en salud de 3 comunidades localizadas periféricamente a la sede del sector, se habla de la comunidad de varillal arriba, también conocida como varillal san pablo, Wally y El Carmen propiamente dicho; con una población total de 4835 habitantes de los cuales 985 son niños menores de 6 años.

Cada comunidad tiene una distancia considerable, de El Carmen a Wally hay aproximadamente $5 \mathrm{~km}$ y al Varillal Arriba $3 \mathrm{~km}$. Con una carretera poco transitable y muy deteriorada principalmente la que conecta con Varillal Arriba, que en tiempos de invierno se vuelve prácticamente intransitable. (INIDE, 2014)

La sede del sector 9, está localizado al lado izquierdo de la carretera que comunica al Naranjo y limita al norte con preescolar atendido por profesora Glenda, al sur con la casa de habitación de doña Agustina, al este con la iglesia evangélica y al oeste con predio vacío. Los actores sociales y/o políticos en el sector son: MINED, GFCV, con líderes iglesias católicas y evangélicas.

En todas las comunidades atendidas por el sector 9, cuentan con servicios básicos como energías eléctrica en un $100 \%$ y se abastecen de agua no potable que llega a los hogares por medio de tubos en casi un $95 \%$ de la población, el resto tienen pozos excavados sin mantenimiento, que ocupan para la realización de los quehaceres y permanecen descubierto, aparte una de las piletas de El Carmen esta descubierta, y esta agua se está contaminando conllevando a aumentar los cuadros diarreicos en la comunidad en todas las edades y también a la producción de vectores que provocan enfermedades febriles y respiratorias. (INIDE, 2014)

\section{Descripción del programa comunitario de salud nutricional (PROCOSAN)}

Dentro de los programas propuestos por el Modelo de Salud Familiar y Comunitario (MOSAFC) en esta unidad asistencial se ejecuta el Programa Comunitario de Salud Nutricional (PROCOSAN), que consiste en fomentar la salud inicialmente en las y los niños de 0 a 6 años, enseñando en las familias hábitos nutricionales y estilos de vida que protegen y fomentan su salud y nutrición. El objetivo de este programa comunitario es mejorar el crecimiento y promover un estado nutricional adecuado en la niñez menor de 6 años 
poniendo énfasis en los menores de 2 años, mediante la ganancia de peso mensual, para lo cual se realizan actividades de peso y talla (MINSA, 2012)

El trabajo que impulsa PROCOSAN se centra en protagonistas, sean éstos personas, familias o comunidades, que construyen socialmente a su propia salud, superando el esquema asistencialista de atención a beneficiarios que esperan una ayuda para mitigar su situación. (MINSA, 2012, pág. 2)

Una estrategia comunitaria nutricional consiste en el fomento de la salud de las personas inicialmente las y los niños, enseñando hábitos nutricionales y estilos de vida que protegen y fomentan su salud y nutrición, se basa en metodologías de trabajo, que contemplan la cultura e idiosincrasia de las diferentes comunidades y proponen cambios de conductas favorables a la salud y el uso de los recursos locales para lograrlo; se centra en protagonistas (individuo, familias y comunidad) que construyen socialmente su propia salud, superando el esquema asistencialista de atención a beneficiarios que esperan una ayuda para mitigar su situación (MINSA, Manual técnico operativo, 2012)

La nutrición comunitaria es un tipo de estrategia que tiene como objetivo mejorar el estado nutricional y de salud de los individuos y grupos de población de una comunidad. Los profesionales que desarrollan su labor en este campo participan en la confección y puesta en marcha de políticas y programas de actuación encaminados a fomentar hábitos alimentarios saludables. Estos tres elementos: grupos de población, políticas alimentarias nutricionales y programas de intervención forman el eje de actuación del trabajo en nutrición comunitaria (Aranceta, 2013)

El programa comunitario de salud y nutrición (PROCOSAN) en nuestro contexto es la única estrategia comunitaria de nutrición; es una nueva forma de hacer nutrición a partir del 2000. Nace luego de una serie de estudios que permitieron documentar que los programas de intervención en nutrición en el país, no estaban dando el impacto necesario, a pesar que el $80 \%$ de los presupuestos de ellos eran para la distribución de los alimentos.

Es una estrategia formulada para niños menores de 60 meses de edad y en el área rural, con problemas nutricionales elevados con el fin de cambiar el comportamiento de las prácticas nutricionales de las madres y padres de niños menores de 5 años. Y lo más importante es que es una estrategia dirigida por un ente comunitario y de fácil aplicación ya sea un brigadista o líder comunitario, supervisado por profesionales de la salud nutricional. (Yllescas Hernández, 2017)

El Equipo de Salud Familiar y Comunitario de este puesto de salud, aplica el programa PROCOSAN, mediante la visita una vez al mes a cada comunidad, los días 8,17 y 25 de cada mes, donde participan las madres de familia, red comunitaria y maestros; posteriormente esta información de peso y talla 
es entregada a final de cada mes, a la coordinadora a nivel municipal, en este caso Lic. Dolores Muñoz, quien a su vez realiza consolidado de todos los puestos de salud que están a cargo de PROCOSAN, y es enviada a nivel departamental.

\section{Características de los niños y niñas atendidos en el centro de salud de la comunidad el Carmen}

El $43.46 \%$ de las y los niños desnutridos pertenecen a las edades de 12 a 23 meses de edad, con menos incidencia en las edades de 48-60 meses con un $2.804 \%$ y un $51.40 \%$ pertenecen al sexo masculino y $48.60 \%$ al sexo femenino.

\section{Estado nutricional y el tratamiento aplicado}

El gráfico 1 muestra que existe una prevalencia de desnutrición crónica del $83.18 \%$ en comparación a la desnutrición aguda con un $16.82 \%$.

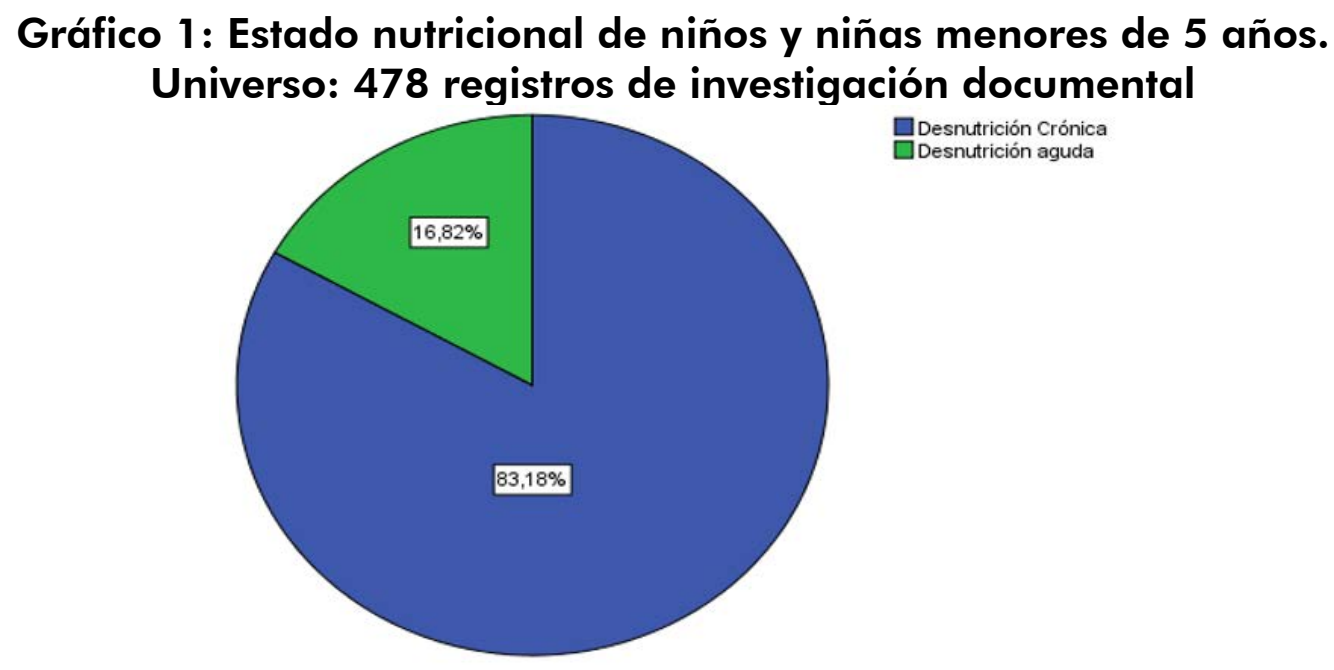

Fuente: Datos secundarios de la investigación documental. Junio 2019

La UNICEF refiere que la desnutrición crónica es un problema de mayor magnitud en cuanto al número de niños afectados, es a veces invisible y recibe menor atención. El retraso en el crecimiento puede comenzar antes de nacer, cuando el niño aún está en el útero de su madre. Si no se actúa durante el embarazo y antes de que el niño y niña cumpla los dos años de edad, las consecuencias son irreversibles y se harán sentir durante el resto de su vida (UNICEF, 2011, pág. 9)

Se puede afirmar que la desnutrición crónica es la que prevalece más por encima de la desnutrición aguda (datos recopilados del censo nutricional realizado de enero a junio del 2019 en san Juan de Rio coco) Lo que hace falta es reforzar actividades que ayuden a disminuir la incidencia de desnutrición crónica y por consiguiente los estragos irreversibles que este problema causa. 


\section{Gráfico 2: Control de sus hijos/as menores de 5 años. Universo de estudio 2014 encuestas}

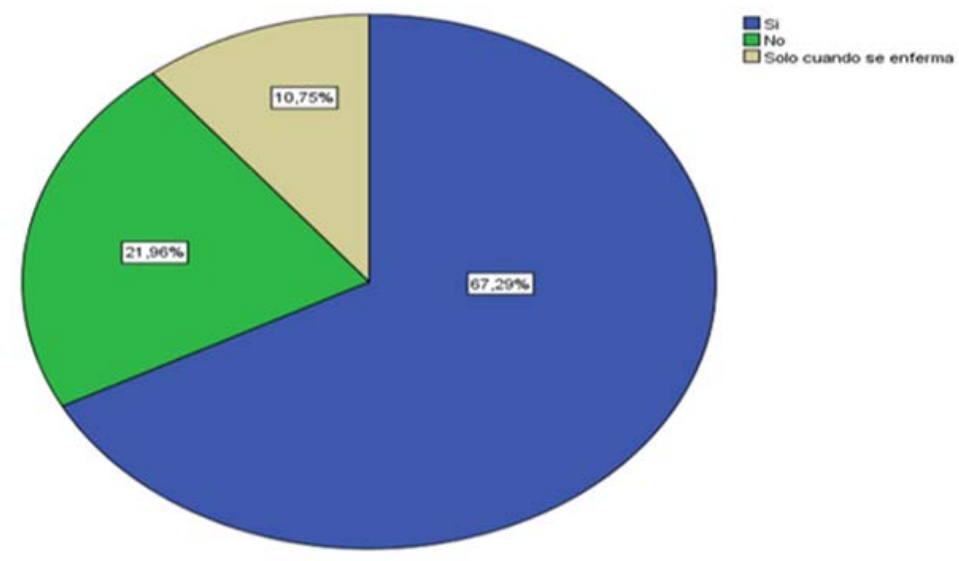

Fuente: Datos primarios de la encuesta. Enero 2020

Gráfico 2: El 67.29\% de las madres encuestadas refieren llevar al niño menos de 5 años al control de Vigilancia y Promoción De Crecimiento y Desarrollo (VPCD), el $21.96 \%$ no acuden al control. Se demuestra que las madres de familia consideran que es importante el control del niño para el conocimiento personal del estado de nutrición del mismo.

Dos madres de familia se refieren a la importancia de llevar a los controles de crecimiento y sesiones de pesaje a su niño:

"Considero que es muy importante traer a mi niño, porque me doy cuenta si ha ganado de peso o no, además me brindan medicamento, charlas sobre alimentación del niño a medida que va creciendo" (Elda Rivera Gómez. Madre de familia, enero 2020)

"Es importante porque lo pesan y lo tallan y si lo ven pálido le dan hierro, aunque a veces no lo traigo porque me queda largo y no tengo para el pasaje" (Maximina Guerrero Moreno. Madre de familia, enero 2020.

En la tabla número 1, se muestra resultados donde las 214 madres encuestadas refieren pesan al niño en cada control. Al consultar a las y los brigadistas sobre ¿Qué materiales le ha facilitado el ministerio de salud?

Tabla 1: Peso al niño/a. Universo de estudio: 214 encuestas

\begin{tabular}{r|r|r|}
\hline Peso & Frecuencia & $\%$ \\
\hline & 214 & $100 \%$ \\
\hline
\end{tabular}

Fuente: Datos primarios de la encuesta, enero 2020

"Bueno, tengo una pesa de calzón para los niños y niñas menores de 2 años y una pesa digital para los mayores de 2 años, por lo cual se pesan 
todos los niños que acuden al programa cada mes" (Yanoris Gómez. Brigadista de El Carmen, enero 2020)

En síntesis, todos los niños y niñas son pesados por la colaboración de los brigadistas de salud y la posesión de materiales para llevar a cabo el pesaje.

En la tabla número 2, se muestra resultados donde las 214 madres encuestadas refieren tallan al niño en cada control.

Tabla 2: Talla al niño/a. Universo de estudio: 214 encuestas

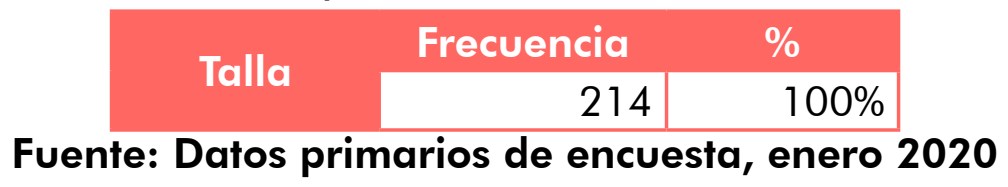

Al consultar a las y los brigadistas sobre ¿̇Qué materiales le ha facilitado el ministerio de salud?

"También, tengo un tallímetro vertical para niños mayores de 3 años y uno horizontal que se pone en la mesa para niños y niñas menores de 3 años, hay un centímetro que se utiliza cuando el niño no se deja pesar" (Yanoris Gómez. Brigadista de El Carmen, enero 2020)

En síntesis, todos los niños y niñas son tallados por la colaboración de los brigadistas de salud y la posesión de materiales para llevar a cabo el tallaje.

El $100 \%$ de los niños y niñas menores de 5 años tienen todas sus vacunas. Según Stekel y Schlesinger (2013) tanto la experiencia clínica como la experimental señalan que la desnutrición y la infección se entrelazan íntimamente potenciando sus efectos en forma recíproca. Es así como en la desnutrición aumenta la incidencia y gravedad de las infecciones y estas últimas al repetirse agravan la desnutrición, determinando un ciclo difícil de quebrar (Stekel \& Schlesinger, 2013)

Realizando un análisis comparativo se muestra que el estado de inmunización evita la presentación de enfermedades en sus formas graves, aun con alto nivel de desnutrición, por tanto, las vacunas son una parte de reforzamiento del sistema inmunológico.

La tabla 3 muestra que el 100\% de los niños se le brindan suplementos de hierro y desparasitante, en contraste con suplementos de Vitamina A que solo se brindan al $46.3 \%$. 
Tabla 3: Entrega de suplementos a niños/as menores de 5 años. Universo de estudio: 214 encuestas

\begin{tabular}{|l|r|r|r|r|r|r|}
\multicolumn{4}{c}{} & \multicolumn{2}{c}{ Hierro } & \multicolumn{2}{c}{ Vitamina A } & \multicolumn{2}{c|}{ Desparasitante } \\
\hline Si & 214 & 100 & 99 & 46.3 & 214 & 100 \\
\hline No & & & 115 & 53.7 & & \\
\hline
\end{tabular}

Fuente: Datos primarios de la encuesta, enero 2020

En definitiva, se facilita hierro y desparasitante a todos los niños menores de 5 años, puesto que son los insumos que más entrada hay en el puesto de salud, no obstante vitamina A se suministra únicamente en jornadas de vacunación anual, por tanto, se confirma con resultados de encuesta aplicada.

La carencia de vitamina $A$ es un problema de salud pública, que según las estimaciones afecta a 190 millones de niños en edad preescolar, los lactantes y los niños pequeños presentan un aumento de las necesidades de vitamina A para hacer frente a su rápido crecimiento y ayudar a combatir las infecciones.

La administración de suplementos de vitamina $A$, a niños de 6 a 59 meses en los países en desarrollo, se asocia a un menor riesgo de mortalidad por todas las causas y una menor incidencia de diarrea (OMS, Administración de suplementos de Vitamina A a lactantes y niños de 6 a 59 meses, 2011, págs. 2-3)

\section{Hábitos alimenticios, nutricionales y de higiene}

En la tabla número 4, se relaciona el tipo de alimentación con la desnutrición de la población en estudio, reflejando que presenta un mayor consumo de origen básico por parte de desnutrición crónica con el $72.9 \%$ diario, mientras que el que menos consumen son alimentos de origen animal con el $68.7 \%$ que no lo consumen. En cuanto a pacientes con desnutrición aguda el alimento mayoritario de consumo es similar a la desnutrición crónica con el 16.9\% y consumen menos alimentos de otros tipos con el $7.5 \%$.

Tabla 4. Desnutrición-Tipo de alimentación. Universo de estudio: 214 encuestas

\begin{tabular}{|c|c|c|c|c|c|c|c|c|c|c|}
\hline & \multicolumn{3}{|c|}{ Origen animal } & \multicolumn{3}{|c|}{ Alimentos básicos } & \multicolumn{3}{|c|}{ Otros alimentos } \\
\hline & & $\begin{array}{c}1 \text { vez } \\
\text { semana }\end{array}$ & $\begin{array}{l}2 \text { veces } \\
\text { semana }\end{array}$ & Nunca & Diario & $\begin{array}{l}4 \text { veces } \\
\text { semana }\end{array}$ & Diario & $\begin{array}{l}4 \text { veces } \\
\text { semana }\end{array}$ & $\begin{array}{l}\text { Menor } \\
\text { de } 3 \\
\text { días por } \\
\text { semana }\end{array}$ & Nunca \\
\hline \multirow{2}{*}{$\begin{array}{l}\text { Desnutrición } \\
\text { crónica }\end{array}$} & $\mathrm{Si}$ & 20 & 11 & 147 & 156 & 22 & 1 & 1 & 112 & 64 \\
\hline & $\%$ & 9.4 & 5.1 & 68.7 & 72.9 & 10.2 & 0.5 & 0.5 & 52.3 & 29.9 \\
\hline \multirow{2}{*}{$\begin{array}{l}\text { Desnutrición } \\
\text { aguda }\end{array}$} & $\mathrm{Si}$ & 21 & 0 & 15 & 36 & 0 & 0 & 0 & 16 & 20 \\
\hline & $\%$ & 9.8 & 0 & 7 & 16.9 & 0 & 0 & 0 & 7.5 & 9.3 \\
\hline \multirow{2}{*}{ TOTAL } & $\mathrm{Si}$ & 41 & 11 & 162 & 192 & 22 & 1 & 1 & 128 & 84 \\
\hline & $\%$ & $19.2 \%$ & $5.1 \%$ & $75.7 \%$ & 89.8 & $10.2 \%$ & $0.5 \%$ & $0.5 \%$ & $59.8 \%$ & 39.2 \\
\hline
\end{tabular}

Fuente: Datos primarios de la encuesta, enero 2020 
Para Ruiz Obando (2010) la disponibilidad y acceso a los alimentos influyen de manera significativa al consumo porque es necesario que el consumidor cuente con los alimentos disponibles y que tenga la capacidad adquisitiva para comprarlo o producirlo; pero su cultura, hábitos alimentarios y demás factores como bajos ingresos económicos familiares determinarán si los consume o no.

Así mismo, también interviene la distribución familiar al momento de repartir los alimentos, se tiene la creencia incorrecta de la niña y/o mujer no necesitan tanto alimento como el niño y el hombre. Se ha documentado que en hogares pobres de América Latina, los hombres reciben un trato preferencial en las comidas, tanto en términos de porciones, repeticiones y alimentos seleccionados (Ruiz Obando, 2010)

La disponibilidad de los alimentos está en relación a bajos ingresos económicos, el mayor consumo es de origen básico (maíz, frijoles) porque la mayoría de estas familias son agricultoras y siembran granos básicos, pero muy poco implementan el cultivo y consumo de legumbres. El acceso a alimentos de origen animal es mucho más difícil, por el precio y raciones que llegan a la comunidad por bajo comercio.

En el gráfico 3 , el $37.38 \%$ de las prácticas alimentarias son deficientes y el $29.91 \%$ son buenas.

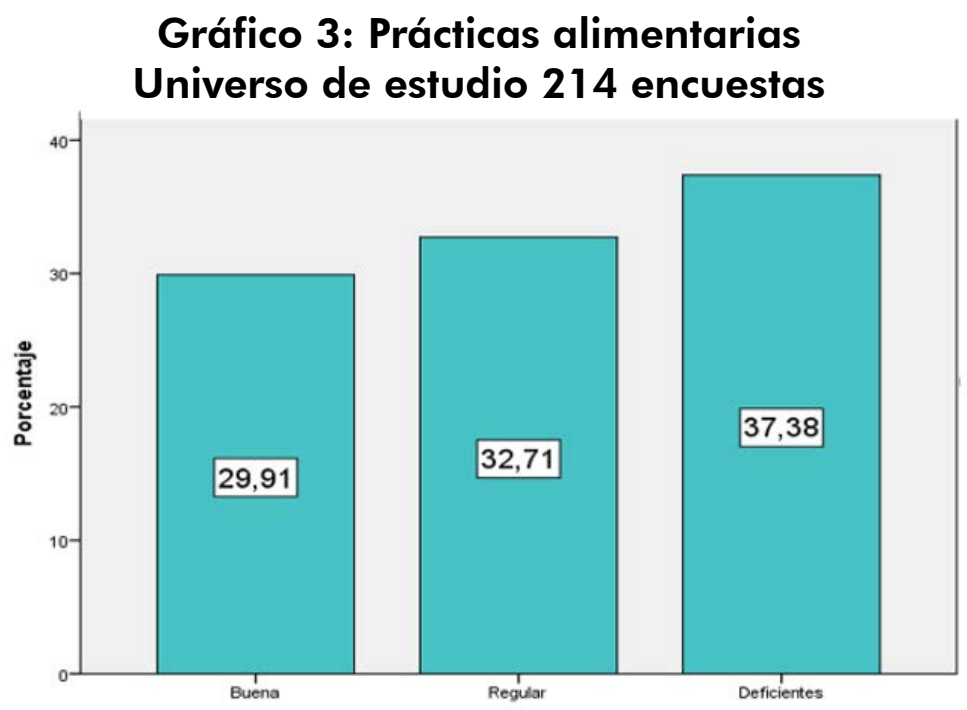

Fuente: Datos primarios de la encuesta, enero 2020

Según Sebba Marino (2015) ciertos factores sociodemográficos incluidos el bajo ingreso económico de las familias están asociados a los patrones de consumo y a cambios de comportamiento alimentario (Sebba Marino, 2015, pág. 37) 
Se determina que las prácticas alimentarias de la población son deficientes debido a comportamientos y prácticas alimentarias inadecuadas.

El Gráfico 4, muestra el 44.86\% las prácticas higiénicas son deficientes, el $31.78 \%$ regular y el $23.36 \%$ es buena.

\section{Gráfico 4: Hábitos de higiene Universo de estudio 214 encuestas}

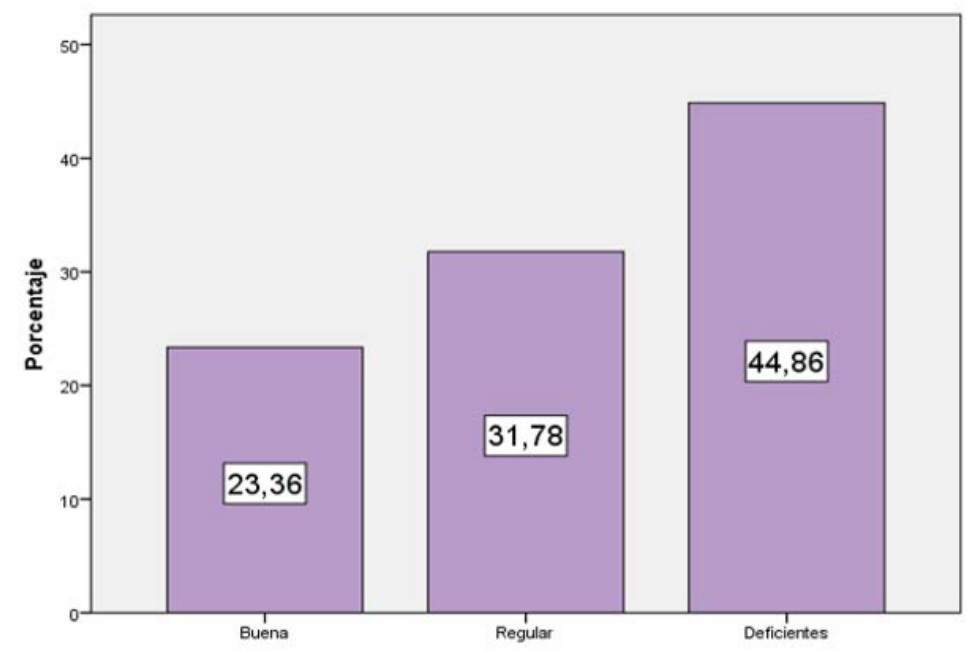

\section{Fuente: Datos primarios de la encuesta, enero 2020}

Se les preguntó a madres sobre: ¿Qué limitaciones tiene para lograr una buena salud e higiene en el hogar? A lo que se encuentra:

"La limitación más evidente es la falta de agua, por tanto, se debe racionalizar bastante porque sólo viene una vez al día" (María Virginia Hernández. Madre de familia, enero 2020)

"por la falta de agua la lavada de la ropa se vuelve más difícil por tanto se trata de cambiar sólo una vez de ropa al día" (Noemí Aguirre Zavala, Madre de familia, enero 2020)

"Lo que se nos dificulta es porque no tenemos agua en el día, sólo viene en la mañana a las 4 am y se va a las 6 am, el tiempo es corto y no se logra llenar muchos recipientes" (María del Carmen Centeno Olivas. Madre de familia, enero 2020)

"A parte de no contar con agua en todo el día esta no es clorada y da bastante dolores de estómagos, de hecho, aunque lavemos los alimentos con ésta agua igual llevan cierta cantidad de bacterias que causan enfermedades" (Hilda Calderón Iglesias. Madre de familia, enero 2020)

Las prácticas de higiene son deficientes por la falta de recursos como el agua, no sólo se carece de este servicio las 24 horas del día, sino que también carece de tratamiento, o cloración. Así que ciertas actividades son deficientes. 


\section{Comprobación de la hipótesis de investigación}

En la investigación se centra en conocer si la calidad de las prácticas alimentarias y hábitos de higiene influyen en la desnutrición de niños y niñas menores de 5 años, para lo cual se crean dos hipótesis.

$\mathrm{H}_{0}$ : A menor calidad de las prácticas alimentarias y de hábitos de higiene, mayor es la incidencia de desnutrición en niños de 0 a 5 años, atendidos en el puesto de salud, el Carmen de San Juan de rio coco.

$\mathrm{H}_{1}$ : La calidad de las prácticas alimentarias y de hábitos de higiene, no influye en la incidencia de desnutrición en niños de 0 a 5 años, atendidos en el puesto de salud, el Carmen de San Juan de Rio Coco.

Gráfico 5 indica que se acepta la hipótesis que dice que A menor calidad de las prácticas alimentarias y de hábitos de higiene, mayor es la incidencia de desnutrición en niños de 0 a 5 años.

\section{Gráfico 5: Prácticas alimentarias - Desnutrición Universo de estudio 214 encuestas}

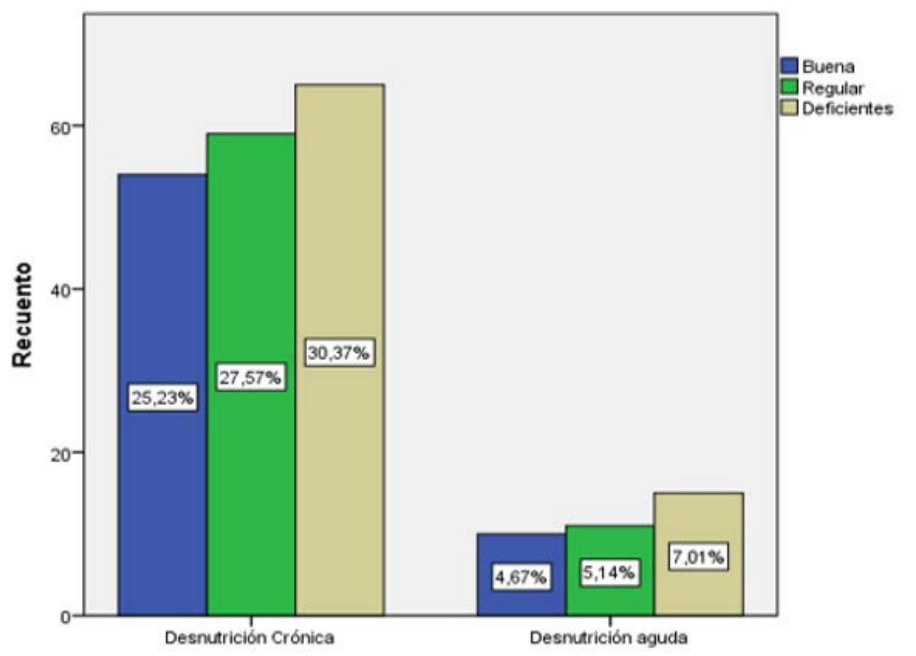

\section{Fuente: Datos primarios de la encuesta, enero 2020}

Gráfico 6 indica que se acepta la hipótesis que dice que A menor calidad de las prácticas alimentarias y de hábitos de higiene, mayor es la incidencia de desnutrición en niños de 0 a 5 años. 


\section{Gráfico 6: Hábitos de higiene - Desnutrición Universo de estudio 214 encuestas}

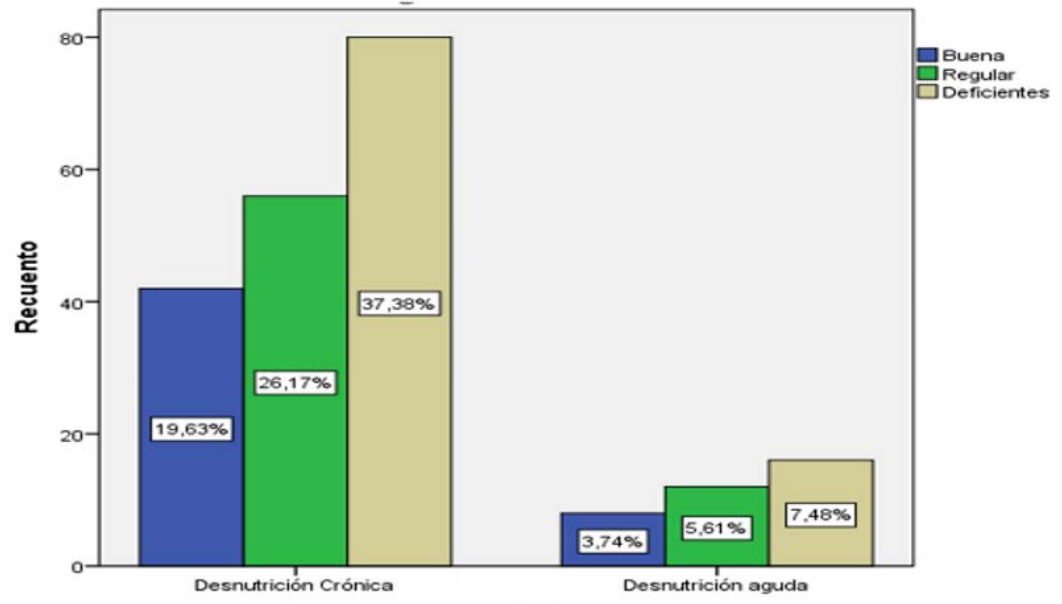

Fuente: Datos primarios de la encuesta, enero 2020

Para elaborar una propuesta de estrategia comunitaria, lo primero fue realizar el análisis de las principales Fortalezas, Oportunidades, Debilidades y Amenazas (FODA) sobre la atención comunitaria del programa PROCOSAN que se realiza del centro de salud El Carmen, y estos fueron los resultados:

\section{Tabla 5: Análisis FODA}

\begin{tabular}{|c|c|c|c|}
\hline Fortalezas & Oportunidades & Debilidades & Amenazas \\
\hline $\begin{array}{l}\text { F.1. Enseña hábitos } \\
\text { nutricionales y estilos } \\
\text { de vida que protegen } \\
\text { y fomentan la salud y } \\
\text { nutrición. } \\
\text { F.2. Contempla } 2 \\
\text { componentes madre e } \\
\text { hijo. } \\
\text { F.3. Brinda atención } \\
\text { de las morbilidades a } \\
\text { nivel de la comunidad. } \\
\text { F.4. Brigadistas } \\
\text { se encuentran } \\
\text { capacitadas para } \\
\text { realización de pesaje y } \\
\text { tallaje de niñas y niños. } \\
\text { F.5. Enfatiza en niñas } \\
\text { y niños menores de } 2 \\
\text { años. }\end{array}$ & $\begin{array}{l}\text { O.1. Es un Programa } \\
\text { elaborado para } \\
\text { abordar el fenómeno } \\
\text { de la desnutrición } \\
\text { desde el marco del } \\
\text { quehacer del MINSA } \\
\text { como la institución } \\
\text { rectora de la salud } \\
\text { y garante de este } \\
\text { derecho en nuestro } \\
\text { país Nicaragua. }\end{array}$ & $\begin{array}{l}\text { D.1. Se centra en } \\
\text { consejería. } \\
\text { D.2. Se basa en el } \\
\text { aprendizaje de los } \\
\text { adultos. } \\
\text { D.3. No incluye } \\
\text { acciones para combatir } \\
\text { la limitación en la } \\
\text { adquisición de los } \\
\text { alimentos. } \\
\text { D.4. No se forma } \\
\text { comité encargado } \\
\text { exclusivamente de } \\
\text { vigilar la nutrición de } \\
\text { los niños. } \\
\text { D.5. No es una } \\
\text { estrategia que } \\
\text { profundiza en } \\
\text { el manejo de } \\
\text { desnutrición. }\end{array}$ & $\begin{array}{l}\text { A. } 1 \text {. Familias no } \\
\text { pueden cumplir con } \\
\text { las orientaciones sobre } \\
\text { hábitos alimenticios } \\
\text { por ser de escasos } \\
\text { recursos. } \\
\text { A.2. Desinterés por } \\
\text { parte de los padres de } \\
\text { acudir a las sesiones } \\
\text { de pesaje. } \\
\text { A.3. Posibilidad de } \\
\text { subregistros en datos } \\
\text { de peso y talla por la } \\
\text { inasistencia de niños y } \\
\text { niñas al programa de } \\
\text { peso y talla. }\end{array}$ \\
\hline
\end{tabular}




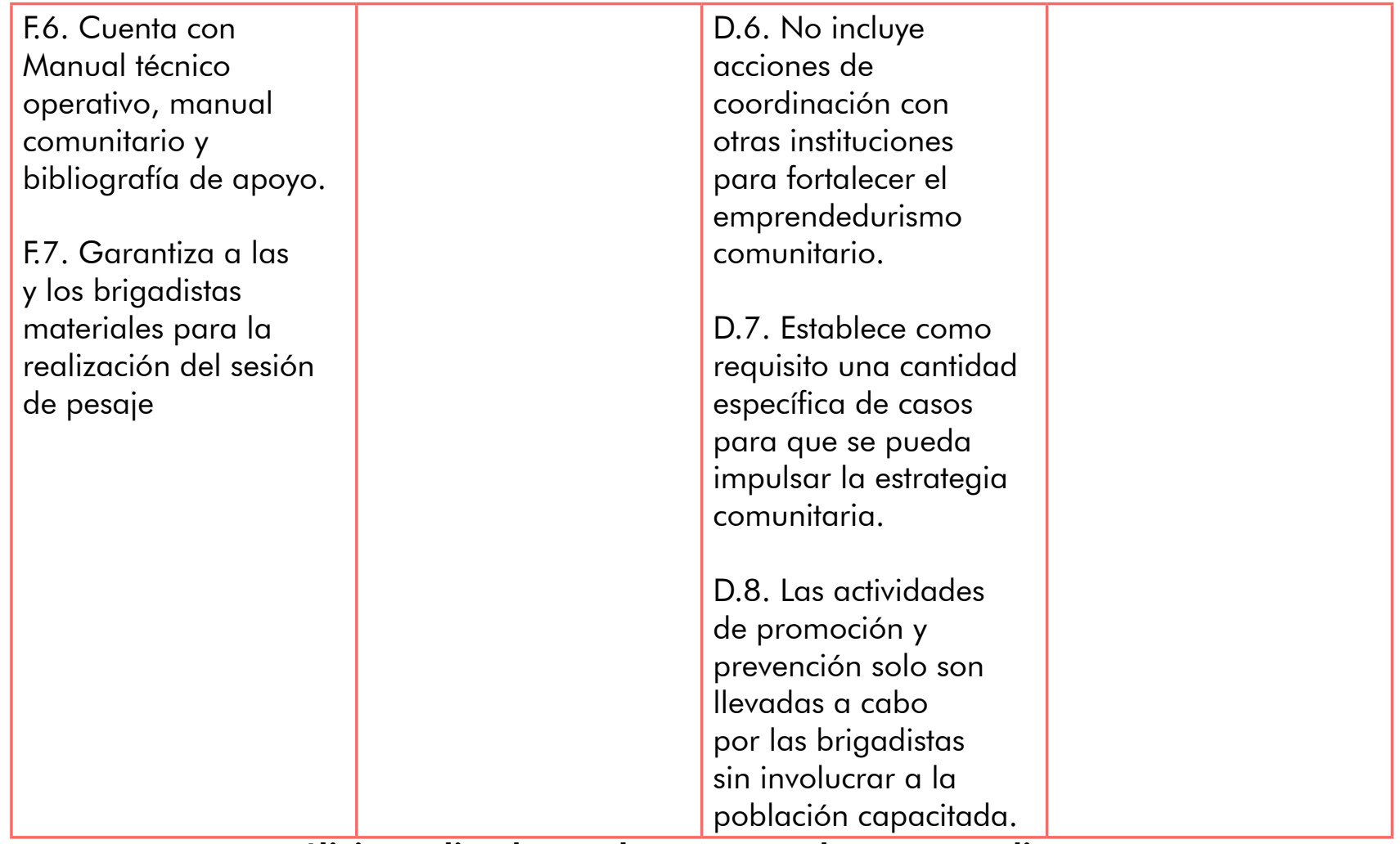

Fuente: Análisis realizado por los autores de este estudio. Enero, 2020.

A partir del análisis anterior, en el marco de este estudio se propone una nueva estrategia comunitaria para la prevención y atención de la desnutrición en la niñez de 0-5 años, denominada ECOPADES, y se describe a continuación:

ECOPADES Es una Estrategia comunitaria para la Prevención y Atención de la desnutrición en la niñez de 0-5 años, la cual está centrada en la participación activa de la familia y comunidad como protagonistas de la restitución del derecho de su propia salud y orientada a contribuir a la erradicación de la desnutrición en la niñez fomentando buenas practicas alimenticias, siendo su objetivo contribuir a la erradicación de la desnutrición en la niñez de 0-5 años en el Sector de El Carmen, San Juan del Rio Coco, Nicaragua.

Esta estrategia está dirigida específicamente a las comunidades del Sector No. 9, denominado El Carmen en el Municipio de San Juan del Río Coco, Nicaragua, pero puede ser implementada en cualquier comunidad donde existan niños de 0-5 años, y que tengan interés en prevenir o reducir la incidencia de la desnutrición. Para el éxito de esta estrategia se requiere del compromiso de las familias, comunidad, y del trabajo coordinado con SILAIS-MINSA-ESAFC, como la institución garante del derecho a la salud en Nicaragua.

Para su implementación, ECOPADES se ha estructurado alrededor de cinco componentes, los cuales incluyen acciones para el trabajo con el Personal del MINSA, familias, comunidad en general y liderazgo, y concretamente con niñez de 0-5 años y mujeres embarazadas.

Parte de los cinco componentes se describen a continuación: 


\section{Componente Núm. 1: capacitación del personal de salud}

El Ministerio de Salud (MINSA) deberá instruir al personal que labora desde el nivel departamental y municipal hasta el nivel sectorial (Puestos de Salud) a fin de que conozcan la Estrategia y la promuevan en los Sectores, haciendo énfasis en objetivos, componentes y metodología a aplicar. Además, se incluye el adiestramiento en el uso de los instrumentos de apoyo y seguimiento a los Comités comunitarios, estructura alrededor de la que funcionará ECOPADES.

Esta capacitación, partirá de un evento a nivel nacional el cual se irá replicando en cada uno de los niveles operativos en los que se organiza el Ministerio de Salud (MINSA) en los territorios, con la posibilidad de generar otros espacios para el fortalecimiento de capacidades del personal a cargo en base a las demandas y necesidades que surjan durante la implementación de la Estrategia.

\section{Componente Núm. 2: organización y participación comunitaria}

Las acciones previstas para promover la organización y participación comunitaria, son las siguientes:

- Presentación de la Estrategia a la comunidad y a Organismos e instituciones que tienen incidencia en dicha localidad.

- Conformación del Comité de Nutrición (COMNUT)

- Divulgación de roles y funciones del Comité de Nutrición.

- Capacitación a miembros del Comité.

- Captación y capacitación de Promotoras-es comunitarios de Nutrición.

- Planificación y evaluación de las actividades del Comité.

\section{Componente Núm. 3: fortalecimiento de capacidades de las familias}

Las actividades contempladas en este componente son las siguientes:

- Identificación de familias con niñez de 0-5 años y mujeres embarazadas en riesgo o en situación de desnutrición.

- Capacitación a las familias: Las capacitaciones a las familias se realizarán una vez al mes.

\section{Componente Núm. 4: gestión de los alimentos}

- Concurso "Producimos y consumimos más y mejores alimentos": A través de este concurso se pretende promover la producción y consumo de alimentos de manera que las familias puedan diversificar su dieta diaria de manera balanceada. Para ello, se lanzará una convocatoria abierta a todas las familias de la comunidad en donde se le darán a conocer los criterios para la participación, y se les instará a inscribirse para lo que deberán ponerse en contacto con él o la Responsable de Gestión Alimentaria (Miembro del Comité de Nutrición). 
- Ferias comunitarias para promover formas de preparación de los alimentos: Cada tres meses el Comité promoverá las Ferias comunitarias de alimentos en donde las familias integradas en la implementación de esta Estrategia puedan vender alimentos preparados utilizando los productos de su JARDIN y en base a las nuevas recetas que han aprendido a partir de las capacitaciones o el intercambio con otras familias. Se espera que las familias elaboren platillos nutritivos y limpios para ofertar a la población promoviendo así el emprendedurismo desde la propia comunidad y fomentando diversificación de los alimentos.

- Creación y funcionamiento del Club de Nutrición Infantil: Los niños y niñas en riesgo o en situación de desnutrición, sobre todo que provengan de familias en situación de vulnerabilidad económica y que no puedan garantizarle una dieta balanceada, tendrán la oportunidad de acceder al servicio de alimentación que brindará el Club de Nutrición Infantil.

\section{Componente Núm. 5: comunicación para el cambio}

- Implementación de Campaña sobre la importancia de la Nutrición.

- Actividades lúdicas con niñas y niños en las Escuelas:

- Elaboración y ubicación de carteles que promueven la nutrición, en puntos estratégicos de la comunidad.

El Ministerio de Salud deberá elegir un encargado a nivel central y en cada municipio, el cual estará encargado de vigilar el cumplimiento de esta Estrategia comunitaria y valorar si ha habido mejoría en el estado nutricional de la niñez menor de 5 años.

La información obtenida de las diferentes actividades orientadas por la estrategia debe seguir un flujo lógico con el fin de que las diferentes instancias manejen las acciones que se están realizando en las comunidades y si responden o no lo indicado en la estrategia y realizar un adecuado monitoreo se describe a continuación en la siguiente ilustración.

Ilustración No.1 Flujo de la información ECOPADES

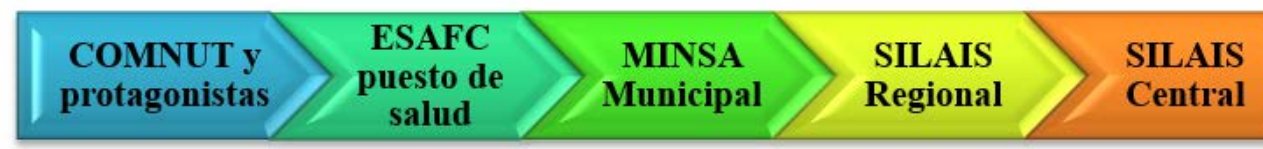

Fuente: Elaborado para este estudio. Enero, 2020.

Además de que se deberá realizar supervisión de la Estrategia, cada mes se deberá enviar un consolidado de las actividades realizadas en la comunidad los cuales servirán además para monitoreo y seguimiento tomando en cuenta lo descrito en la estrategia. 


\section{CONCLUSIONES}

El estudio revela la prevalencia de la desnutrición crónica por encima de la aguda. Con la información recabada en el estudio se confirma la hipótesis de que a menor calidad de las prácticas alimentarias y de hábitos de higiene, mayor es la incidencia de desnutrición en niños de 0 a 5 años, atendidos en el puesto de salud El Carmen, San Juan de Rio Coco, dicha hipótesis se cumple en un $84.4 \%$.

En cuanto a la atención en salud los padres consideran como buena y menos del $1 \%$ como mala a pesar de que en el tratamiento solo se den suplementos de hierro y desparasitante en cada control ya que la vitamina $A$ se administra únicamente en jornada. Se evidencia una asistencia significativa a las sesiones comunitarias de peso y talla que se realizan mensualmente en el sector.

Un hallazgo significativo es que las familias no están incorporando a la dieta alimentos ricos en proteínas y vitaminas, se centran sólo en alimentos con altos contenidos de carbohidratos porque los modelos de producción están limitados al cultivo tradicional del café y granos básicos, todo esto muestra prácticas alimentarias y nutricionales deficientes.

Se determina que las prácticas alimentarias y prácticas de higiene de la población son deficientes debido a comportamientos y prácticas alimentarias inadecuadas y por la falta de recursos como el agua, no sólo se carece de este servicio las 24 horas del día, sino que también carece de tratamiento, o cloración, respectivamente.

ECOPADES una propuesta de Estrategia Comunitaria Para la Prevención y Atención de la Desnutrición en la niñez de 0-5 años, que surge a través de análisis FODA del programa comunitario de salud y nutrición y se propone a las autoridades competentes.

\section{BIBLIOGRAFÍA}

Bamba, X., Espinoza, D., \& Fajardo, V. (2010). Prevalencia de la mala nutricion y su relacion con el rendimiento academico en niños de la escuela Julio Matovelle. Periodo lectivo 2008-2010. Universidad de Cuenca, Facultad de Ciencias Médicas. Cuenca, Ecuador: Universidad de Cuenca. Recuperado el 29 de 12 de 2019, de https://dspace.ucuenca.edu.ec/ bitstream/123456789/3516/1/MED22.pdf 
Barahona, P., \& Barahona, J. (2012). Habitos de Higiene en el estudiante y su desarrollo mediante la aplicacion de instructivo. Universidad Estatal de Milagro (UNEMI). Ecuador: UNEMI. Obtenido de http://repositorio.unemi. edu.ec/bitstream/1 23456789/1947/1/Los\%20h\%C3\%A 1 bitos\%20 de\%20higiene\%20en\%20el\%20estudiante\%20y\%20su\%20desarrollo\%20 mediante\%20la\%20aplicacion\%20de\%20instructivo..pdf

INIDE. (2014). Situacion sociodemográfica San Juan de Rio - Coco. Managua. Jansen, H., Rodriguez, À., Damon, A., \& Pender, J. (2003). Determinantes de estrategias comunitarias de subsistencia y el uso de prácticas conservacionistas de producción agrícola en las zonas de ladera en Honduras. Instituto Internacional para la Investigación de Políticas Alimentarias (IFPRI). Honduras: EPTD. Obtenido de http://ebrary.ifpri. org/utils/getfile/collection/p15738coll2/id/75880/filename/75881.pdf

Lopez Roldan, P., \& Fachelli, S. (2015). Metodologia de la Investigación Social Cuantitativa. Barcelona, España: UAB .

MINSA. (2009). Normas de alimentación y nutrición para embarazadas, puerperas y niñez menor de 5 años. Managua, Nicaragua: MINSA. Recuperado el 14 de 12 de 2019, de https://siteal.iiep.unesco.org/sites/ default/files/sit_accion_files/ni_0022_0.pdf

MINSA. (2012). Manual técnico operativo de la niñez (PROCOSAN). Managua: MINSA.

OMS. (2011). Administración de suplementos de Vitamina A a lactantes y niños de 6 a 59 meses. Obtenido de Administración de suplementos de Vitamina A a lactantes y niños de 6 a 59 meses.: https://apps. who.int/iris/bitstream/handle/10665/44721/9789243501765_spa. pdf;isessionid = BCC971 A07B5076F503A149B2EE2C6E51 ? sequence $=1$

OMS. (31 de Agosto de 2018). Obtenido de https://www.who.int/es/news$\mathrm{room} /$ fact-sheets/detail/healthy-diet

Ruiz Hurtado, S. (2018). Estado nutricional, hábitos alimentarios y prácticas de actividad fisica de niños de 6 a 11 años que pertenecen a la asociación de padres de niños y jóvenes diabéticos de nicaragua (APNJDN). Septiembre 2016-marzo 2018. UNAN-Managua. Managua, Nicaragua: UNAN Managua. Obtenido de https://repositorio.unan.edu.ni/9756/1/98946. pdf

Ruiz Obando, A. d. (2010). Seguridad alimentaria y nutricional de las familias rurales de las comarcas: Los 24, las cortezas y la montañita número 2 del departamento de Masaya, municipio Tisma. Noviembre 2009-Junio 2010. Universidad Nacional Agraria (UNA), Facultad de Desarrollo Rural. Managua: Universidad Nacional Agraria (UNA). Obtenido de https:// repositorio.una.edu.ni/818/1/tne10r934.pdf

Sebba Marino, M. C. (2015). Prácticas alimentarias y razones para cambios en la alimentación de la población adulta de Brasilia. Revista Cubana de salud pública, Habana-Cuba.

Soriano del Castillo, J. M. (2011). Nutrición básica humana (Vol. 1). (U. d. Valencia, Ed.) Valencia, España: Universidad de Valencia. Recuperado el 13 de 12 de 2019, de https://books.google.com.ni/books?id=-C43kg7

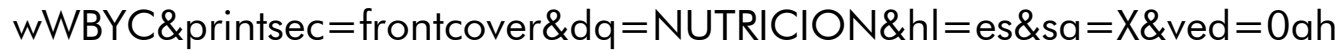


UKEwjSmtWe3LXmAhWi2FkKHdSABZkQ6AEIJzAA\#v=onepage\&q=NUT $\mathrm{RICION} \& \mathrm{f}=$ false

UNICEF. (2011). La desnutrición infantil, causas, consecuencias y estrategias para su prevencion y tratamiento (Vol. 1). (UNICEF, Ed.) Madrid, Madrid, España: UNICEF. Obtenido de https://www.unicef.es/sites/unicef.es/files/ comunicacion/Informe_La_desnutricion_infantil.pdf

Vargas Cordero, Z. R. (2017) $)$. La investigación aplicada: Una formade conocer las realidades con evidencia científica. (U. d. Rica, Ed.) REVISTA EDUCACIÓN, 33(1), 155-165. Obtenido de https://www.redalyc.org/ pdf/440/44015082010. pdf? fbclid=IwAR3k1CsY2e5oCkuhJPO

Zambrana, E. (2018). Investigación Documental. Pub Med, 5-6. 